\title{
MÉTODOS ALTERNATIVOS DE ESTIMATIVA DA EVAPOTRANSPIRAÇÃO DE REFERÊNCIA ANUAL E NAS DIFERENTES ESTAÇÕES DO ANO EM CURITIBA-PR, BRASIL
}

\author{
Bruno César Gurski ${ }^{1}$, Jorge Luiz Moretti de Souza ${ }^{2}$, Daniela Jerszurki ${ }^{3}$, Rossana Ferrari \\ Schäfer ${ }^{4}$, Herbert Schäfer ${ }^{5}$
}

\footnotetext{
${ }^{1}$ Doutorando em Ciência do Solo, Departamento de Solos e Engenharia Agrícola, Universidade Federal do Paraná (UFPR), Curitiba-PR, Brasil. E-mail: brunocep@gmail.com

${ }^{2}$ Professor, Departamento de Solos e Engenharia Agrícola, UFPR, Curitiba-PR, Brasil

${ }^{3}$ Post-doc BGU, Jacob Blaustein Institutes for Desert Research, Ben-Gurion University of the Negev, Sede Boqer Campus, Israel

${ }^{4}$ Mestre em Ciência do Solo, Departamento de Solos e Engenharia Agrícola, UFPR, Curitiba-PR, Brasil.

${ }^{5}$ Professor Departamento de Informática, Universidade Tuiuti do Paraná, Curitiba-PR, Brasil
}

RESUMO: Curitiba-PR é um grande centro consumidor de alimentos, sua região metropolitana produz hortifrutigranjeiros durante o ano inteiro e quase a totalidade dos agricultores utiliza irrigação, sendo esse o principal custo da produção. No entanto, em muitos locais, por serem de difícil acesso, há carência de dados climáticos para obtenção da evapotranspiração de referência (ETo) por modelos consagrados, gerando a necessidade de testar modelos alternativos para essa estimativa que é tão importante para determinar a demanda hídrica vegetal na região, diminuindo os custos de produção e maximizando o uso eficiente da água. Teve-se como objetivo no presente trabalho avaliar cinco métodos alternativos (Thornthwaite, Camargo, Hargreaves e Samani, Budyko e Linacre) de estimativa da ETo em relação ao método de Penman-Monteith em Curitiba-PR, anualmente e nas diferentes estações do ano. Os dados climáticos compreenderam o período de 1998 a 2007. Os dados foram analisados por meio do coeficiente de correlação $(\mathrm{R})$, índices de concordância e desempenho, e estimativas dos erros. O método de Camargo, seguido de Thornthwaite, demonstram melhor desempenho para estimativa anual da ETo em Curitiba-PR, necessitando pequena quantidade de dados de entrada para uma estimativa adequada. Considerando as estações do ano, os melhores métodos para o verão são Linacre, Camargo e Thornthwaite, para o outono é Camargo, para o inverno são Camargo, Linacre e Thornthwaite, e para a primavera são Linacre e Camargo. Os métodos de Hargreaves e Samani, e Budyko são inadequados para estimar a ETo para o tipo climático $\mathrm{Cfb}$ na região de Curitiba-PR.

Palavras-chave: Demanda hídrica. Modelos simplificados. Consumo hídrico. Irrigação. Penman Monteith.

\section{ALTERNATIVE METHODS OF ESTIMATING THE ANNUAL REFERENCE EVAPOTRANSPIRATION AND IN DIFFERENT SEASONS IN CURITIBA-PR, BRAZIL}

\begin{abstract}
Curitiba-PR is a major consumer food center, its metropolitan area produces vegetables throughout the year and almost all farmers use irrigation, which is the main cost
\end{abstract}

Cultura Agronômica, Ilha Solteira, v.25, n.2, p.155-166, 2016 
of production. However, in many places, because they are difficult to access, there is a lack of climate data to obtain the reference evapotranspiration (ETo) by renowned models, creating the need to test alternative models for this estimate that is so important to determine vegetable water demand in the region, reducing costs and maximizing the efficient use of water. This study was aimed to evaluate five alternative methods (Thornthwaite, Camargo, Hargreaves and Samani, Budyko and Linacre) to estimate the ETo in relation to the Penman-Monteith method, in Curitiba-PR, annually and in different seasons. Estimates of ETo were compared with the standard daily ETo calculated with the method of Penman Monteith. Climatic data were collected in Curitiba-PR, from 1998 to 2007. Data were analyzed using correlation coefficient (R), concordance and performance indexes, and estimates of errors. The Camargo method, followed by Thornthwaite, show better performance for annual estimation of the ETo in Curitiba-PR, needing small amount of input data for an adequate estimate. Considering the seasons, the best methods for summer are Linacre, Camargo and Thornthwaite, for autumn is Camargo, for the winter are Camargo, Linacre and Thornthwaite, and the spring are Linacre and Camargo. The methods of Hargreaves and Samani, and Budyko are inadequate to estimate the ETo for climate type Cfb in Curitiba-PR region.

Key words: Water demand. Simplified models. Water consumption. Irrigation. Penman Monteith.

\section{INTRODUÇÃO}

A evapotranspiração de referência (ETo) tem papel fundamental no estudo e determinação das relações hídricas em atividades da engenharia rural, sendo uma das variáveis hidrológicas mais importantes para o cálculo da evapotranspiração da cultura, estimativa e interpretação de balanços hídricos agrícolas, manejo de irrigação e eficiência no uso da água (XU; SINGH, 2005). A ETo pode ser medida com lisímetros e evapotranspirômetros ou estimada por modelos teóricos embasados no clima, solo e planta. Sediyama (1987) relatou a existência de mais de cinquenta métodos de estimativa da ETo, já Pereira et al. (1997) detalharam dezessete métodos e os classificou como empírico, aerodinâmico, balanço de energia, combinado e correlação.

Dentre os principais métodos, o de Penman-Monteith (ALLEN et al., 1998) é um dos mais utilizados atualmente (SOUZA et al., 2014). Apesar da consagração deste método, no Brasil os dados exigidos são de difícil obtenção, devido à diversidade regional (microrregiões especializadas e macrorregiões sem estudos), e a inexistência de séries históricas de dados e equipamentos de medição. Adicionalmente, a instalação de estações meteorológicas é limitada por fatores técnicos e econômicos, bem como dificuldades de interpretar os dados que criam uma série de limitações para simulação hídrica e o planejamento agrícola. Por isso, métodos alternativos que não exijam dados complexos podem superar essas limitações e fornecer um valor razoável de ETo para regiões onde não há estudo algum (PRAVEENA, 2012). Isso representaria um avanço na agropecuária e silvicultura brasileiras, principalmente na agricultura familiar de subsistência que carece de dados neste sentido.

Cultura Agronômica, Ilha Solteira, v.25, n.2, p.155-166, 2016 
A cidade de Curitiba-PR é um grande centro consumidor de alimentos. Sua região metropolitana possui o chamado cinturão verde, formado por pequenos agricultores familiares que produzem a maior parte dos hortifrutigranjeiros da região durante o ano inteiro e quase a totalidade dos agricultores utiliza irrigação, sendo esse o principal custo da produção. No entanto, em muitos locais, por serem de difícil acesso, há carência de dados climáticos para a obtenção da ETo por modelos consagrados, gerando a necessidade de testar modelos alternativos para essa estimativa que é tão importante para saber a demanda hídrica vegetal na região, visto que há produção o ano inteiro, diminuindo os custos de produção e maximizando o uso eficiente da água (SOUZA et al., 2014; COMEC, 2015).

Syperreck et al. (2008) e Souza et al. (2014) estabeleceram que os métodos de estimativa da evapotranspiração são desenvolvidos em condições climáticas específicas, segundo a disponibilidade de dados e a realidade física do local, o que torna necessária a verificação da tendência de cada método na região para a qual venha a ser aplicado. Por isso, para microrregiões especializadas no cultivo de determinadas culturas, a obtenção de modelos alternativos é uma excelente forma de realizar estimativas sobre determinado fenômeno, baseando-se no conhecimento de poucas variáveis descritoras, para que a informação alcance o maior número de pessoas possível.

Diante deste contexto, objetivou-se com o presente estudo avaliar cinco métodos alternativos (Thornthwaite, Camargo, Hargreaves e Samani, Budyko e Linacre) de estimativa da evapotranspiração de referência (ETo) em relação ao método de Penman-Monteith em Curitiba-PR, anualmente e nas diferentes estações do ano.

\section{MATERIAL E MÉTODOS}

O município de Curitiba-PR possui clima Cfb, segundo a classificação de Köppen, predominantemente temperado, relevo levemente ondulado e altitude média de 934,6 m, localizada nas coordenadas $25^{\circ} 25^{\prime} 48^{\prime \prime S}, 4^{\circ} 16^{\prime} 15^{\prime \prime} \mathrm{W}$. Possui precipitação média de $1500 \mathrm{~mm}$ $\mathrm{ano}^{-1}$ (MAACK, 2002).

Os dados climáticos necessários para as estimativa da ETo foram obtidos em estação meteorológica automática localizada nas coordenadas 25²6’03”S, 49¹3'49” W e $935 \mathrm{~m}$ de altitude. Os dados foram fornecidos pelo Instituto Meteorológico do Paraná (SIMEPAR) e compreenderam o período entre 01 de janeiro de 1998 a 31 de dezembro de 2007 (10 anos). Foram necessários dados diários de temperaturas máxima $\left(T_{m a x}\right)$, média $\left(T_{m e d}\right)$ e mínima $\left(T_{m i n}\right)$ diárias do ar; velocidade $\left(U_{z}\right)$ e altura $(z)$ da medida do vento. O número médio de horas de insolação $(n)$ foi retirado de MAPA (1992).

A ETo diária padrão foi estimada com o método de Penman-Monteith (ALLEN et al., 1998):

$$
E T o_{P M i}=\frac{0,408 \cdot \Delta \cdot(R n-G)+\gamma \cdot \frac{900}{\left(T_{M E D}+273\right)} \cdot U_{2} \cdot(e s-e a)}{\Delta+\gamma \cdot\left(1+0,34 \cdot U_{2}\right)}
$$

Cultura Agronômica, Ilha Solteira, v.25, n.2, p.155-166, 2016 
Sendo: EToPM - evapotranspiração de referência estimada com o método de PenmanMonteith no $i$-ésimo dia $\left(\mathrm{mm} \mathrm{dia}^{-1}\right) ; \Delta$ - declividade da curva de pressão de vapor da água à temperatura do ar $\left(\mathrm{kPa}^{\circ} \mathrm{C}^{-1}\right) ; R n$ - radiação líquida na superfície $\left(\mathrm{MJ} \mathrm{m}^{-2} \mathrm{~d}^{-1}\right) ; G$ - balanço do fluxo de calor no solo $\left(\mathrm{MJ} \mathrm{m}^{-2} \mathrm{~d}^{-1}\right) ; \gamma$ - constante psicrométrica $\left(\mathrm{kPa}^{\circ} \mathrm{C}^{-1}\right) ; T_{M E D}-$ temperatura média do ar $\left({ }^{\circ} \mathrm{C}\right) ; U_{2}$ - velocidade do vento a dois metros de altura $\left(\mathrm{m} \mathrm{s}^{-1}\right)$; es tensão de saturação de vapor $(\mathrm{kPa})$; $e a$ - tensão parcial de vapor $(\mathrm{kPa})$.

A velocidade do vento a $2 \mathrm{~m}$ de altura foi obtida a partir da velocidade do vento medida a $10 \mathrm{~m}$ de altura (ALLEN et al., 1998):

$$
u_{2}=u_{z} \cdot \frac{4,87}{\ln (67,8 \cdot z-5,42)}
$$

Sendo: $u_{2}$ - velocidade do vento a dois metros de altura $\left(\mathrm{m} \mathrm{s}^{-1}\right) ; u_{z}$ - velocidade do vento medida a dez metros de altura $\left(\mathrm{m} \mathrm{s}^{-1}\right) ; z$ - altura de medida $(\mathrm{kPa})$.

Os métodos alternativos de estimativa da ETo consistiram em modelos empíricos e simplificados que consideram dados de fácil obtenção como temperatura do ar e a radiação solar incidente, sendo: Thornthwaite (1948), Camargo (1971), Hargreaves e Samani (1985), Linacre (1977) e Budyko (1974). Esses métodos foram escolhidos pois a literatura demonstra que esses são os que mais se adequam ao tipo climático de Curitiba-PR.

Método de Thornthwaite (1948):

A estimativa da ETo considerou um mês de 30 dias e 12 horas de fotoperíodo, seguindo o procedimento adotado por Souza et al. (1994), considerando a temperatura média diária do $\operatorname{ar}\left(T_{M E D} i\right)$.

$$
\begin{aligned}
& \operatorname{ETo}_{T W i}=\frac{N_{i}}{12} \cdot \frac{1}{30} \cdot 16 \cdot\left(\frac{10 \cdot T_{M E D i}}{I}\right)^{a}, \quad \text { sendo: } T_{M E D i}>0^{o} C \\
& a=6,75 \cdot 10^{-7} \cdot I^{3}-7,71 \cdot 10^{-5} \cdot I^{2}+1,7912 \cdot 10^{-2} \cdot I+0,49239 \\
& I=\sum_{i=1}^{12}\left(0,2 \cdot T_{m}\right)^{1,514}, \quad \text { sendo: } T_{m}>0^{o} C
\end{aligned}
$$

Sendo: ЕТотші - evapotranspiração de referência estimada com o método de Thornthwaite (1948) para o $i$-ésimo dia $\left(\mathrm{mm} \mathrm{dia}^{-1}\right) ; N_{i}$ - fotoperíodo do $i$-ésimo dia (horas); $T_{M E D ~} i-$ temperatura média do ar no $i$-ésimo dia $\left({ }^{\circ} \mathrm{C}\right) ; a$ - função cúbica do índice de calor $(I)$ da região (adimensional); $I$ - índice de calor da região (adimensional); $T_{m}$ - temperatura média normal do $m$-ésimo mês do ano $\left({ }^{\circ} \mathrm{C}\right)$.

Método de Camargo (1971):

$$
E_{T M} o_{C M}=Q o_{i} . F . T_{M E D} i
$$

Sendo: ETOCM $_{i}$ - evapotranspiração de referência estimada com o método de Camargo (1971) para o $i$-ésimo dia $\left(\mathrm{mm} \mathrm{dia}^{-1}\right) ; Q o_{i}$ - radiação solar no topo da atmosfera do $i$-ésimo dia, expressa em equivalente de evaporação $\left(\mathrm{mm} \mathrm{dia}^{-1}\right) ; F$ - fator de ajuste que varia com a temperatura média anual do local (como a região de Curitiba possui temperatura média anual 
inferior a $23{ }^{\circ} \mathrm{C}$, o valor de $F$ utilizado foi igual a 0,01$) ; T_{M E D ~}$ - temperatura média do ar no $i$-ésimo dia $\left({ }^{\circ} \mathrm{C}\right)$.

A transformação dos valores de radiação solar no topo da atmosfera do $i$-ésimo dia em equivalente de evaporação $\left(Q o_{i}-\mathrm{mm} \mathrm{dia}^{-1}\right)$ foi realizada a partir da relação:

$$
Q o_{i\left(m m \cdot d i a^{-1}\right)}=\frac{R_{a}}{2,45}
$$

Sendo: $Q o_{i\left(\mathrm{~mm} \mathrm{dia}^{-1}\right)}$ - radiação solar no topo da atmosfera do $i$-ésimo dia em equivalente de evaporação $\left(\mathrm{mm} \mathrm{dia}^{-1}\right) ; R_{a}$ - radiação solar no topo da atmosfera do $i$-ésimo dia $\left(\mathrm{MJ} \mathrm{m}^{-2} \mathrm{dia}^{-}\right.$ $1)$.

Método de Hargreaves e Samani (1985):

$$
\text { EToHS } i=0,0023 \cdot Q o_{i} \cdot\left(T_{M E D i}+17,8\right) \cdot\left(T_{M A X i}-T_{M I N i}\right)^{0,5}
$$

Sendo: EToHS - evapotranspiração de referência estimada com o método de Hargreaves e Samani (1985) para o $i$-ésimo dia $\left(\mathrm{mm} \mathrm{dia}^{-1}\right) ; Q o_{i}$ - radiação solar extraterrestre do $i$-ésimo dia expressa em equivalente de evaporação $\left(\mathrm{mm} \mathrm{dia}^{-1}\right) ; T_{M E D}{ }^{-}$- temperatura média do ar no $i$-ésimo dia $\left({ }^{\circ} \mathrm{C}\right) ; T_{M A X}{ }$ - temperatura máxima do ar no $i$-ésimo dia $\left({ }^{\circ} \mathrm{C}\right) ; T_{M I N} i$ - temperatura mínima do ar no $i$-ésimo dia $\left({ }^{\circ} \mathrm{C}\right)$.

Método de Budiko (1974):

$$
E{ }_{B K} i=0,20 \cdot T_{M E D i}
$$

Sendo: $E T o_{B K}$ - evapotranspiração de referência estimada com o método de Budyko para o $i$-ésimo dia $\left(\mathrm{mm} \cdot \mathrm{dia}^{-1}\right) ; T_{M E D} i$ - temperatura média do ar no $i$-ésimo dia $\left({ }^{\circ} \mathrm{C}\right)$.

Método de Linacre (1977):

$$
E T o_{L C i}=\frac{700 \frac{T_{M E D i}+0,006 z}{100-\varphi}+15\left(T_{M E D i}-T d_{i}\right)}{80-T_{M E D i}}
$$

Sendo: $E T o_{C L}$ - evapotranspiração de referência estimada com o método de Linacre para o $i$-ésimo dia $\left(\mathrm{mm} \cdot \operatorname{dia}^{-1}\right) ; T_{M E D} i$ - temperatura média do ar no $i$-ésimo dia $\left({ }^{\circ} \mathrm{C}\right) ; z$ - altitude do local (m); $\varphi$ - latitude do local (graus); $T d_{i}$ - temperatura de ponto de orvalho no $i$-ésimo dia $\left({ }^{\circ} \mathrm{C}\right)$.

A comparação dos valores de ETo padrão, obtida pelo método de Penman-Monteith, com os valores estimados com os métodos alternativos foi realizada empregando o coeficiente de correlação (R) e sua classificação segundo Hopkins (2000) (Tabela 1). Para avaliar o grau de exatidão entre valores de ETo padrão e os demais métodos estudados, foi utilizado o índice " $d$ " de concordância de Willmott et al. (1985), e como parâmetro estatístico de comparação, utilizou-se o índice " c" adotado por Camargo e Sentelhas (1997), que serve como indicador de desempenho dos métodos alternativos (Tabela 1). Por fim, foram obtidos os erros cometidos em relação à ETo padrão.

Cultura Agronômica, Ilha Solteira, v.25, n.2, p.155-166, 2016 


$$
d=1-\left[\frac{\sum_{i=1}^{n}\left(E_{i}-O_{i}\right)^{2}}{\sum_{i=1}^{n}\left(\left|E_{i}-\bar{O}_{i}\right|+\left|O_{i}-\bar{O}_{i}\right|\right)^{2}}\right]
$$

Sendo: $d$ - índice de concordância de Willmott et al. (1985) (adimensional); $E_{i}$ - valor estimado no $i$-ésimo dia $\left(\mathrm{mm} \mathrm{dia}^{-1}\right) ; O_{i}$ - valor observado no $i$-ésimo dia $\left(\mathrm{mm} \mathrm{dia}^{-1}\right) ; \bar{O}_{i}-$ média da variável observada no período considerado $\left(\mathrm{mm} \mathrm{dia}^{-1}\right)$.

$$
c=|R \cdot d|
$$

Sendo: $c$ - índice “c” de desempenho proposto por Camargo e Sentelhas (1997) (adimensional); $R$ - coeficiente de correlação (adimensional) obtido na regressão (adimensional).

$$
\begin{aligned}
& E A M=\frac{1}{n} \cdot \sum_{i=1}^{n}\left(\left|E_{i}-O_{i}\right|\right) \\
& R M S E=\sqrt{\frac{1}{n} \cdot \sum_{i=1}^{n}\left(E_{i}-O_{i}\right)^{2}}
\end{aligned}
$$

Sendo: $E A M$ - erro absoluto médio $\left(\mathrm{mm} \mathrm{dia}^{-1}\right) ; R M S E$ - raiz quadrada media do erro (mm

\begin{tabular}{|c|c|c|c|}
\hline \multirow{2}{*}{ Intervalo } & \multirow{2}{*}{ Classificação } & Intervalo & Desempenho \\
\hline & & $c>0,85$ & Ótimo \\
\hline $1,0<\mathrm{R} \leq 0,9$ & Quase Perfeita & $0,85 \leq \mathrm{c}<0,75$ & Muito Bom \\
\hline $0,9<\mathrm{R} \leq 0,7$ & Muito Alta & $0,75 \leq \mathrm{c}<0,65$ & Bom \\
\hline $0,7<\mathrm{R} \leq 0,5$ & Alta & $0,65 \leq \mathrm{c}<0,60$ & Mediano \\
\hline $0,5<\mathrm{R} \leq 0,3$ & Moderada & $0,60 \leq \mathrm{c}<0,50$ & Sofrível \\
\hline $0,3<\mathrm{R} \leq 0,1$ & Baixa & $0,50 \leq \mathrm{c}<0,40$ & Mau \\
\hline $0,1<\mathrm{R} \leq 0,0$ & Muito Baixa & $\mathrm{c} \leq 0,40$ & Péssimo \\
\hline
\end{tabular}
$\left.\operatorname{dia}^{-1}\right) ; n$ - número de observações.

Tabela 1. Classificação do coeficiente de correlação (R) segundo Hopkins (2000) e desempenho do índice "c" de Camargo e Sentelhas (1997).

\section{RESULTADOS E DISCUSSÃO}

A estatística descritiva dos dados climáticos utilizados para Curitiba-PR demonstrou elevada amplitude térmica ao longo do dia, umidade relativa alta, irregularidades na velocidade do vento e estações climáticas bem definidas ao longo do ano (Tabela 2). Esses fatores influenciam diretamente nos métodos alternativos de estimativa da ETo.

Os métodos de Thornthwaite e Camargo apresentaram valores médios de ETo próximos daqueles estimados com a metodologia padrão (EToPM) (Figura 1). Os métodos de Thornthwaite e Camargo também apresentaram bom desempenho para o interior paulista que 
possui condições subtropicais úmidas, quando comparado a dados obtidos em evapotranspirômetros (CAMARGO; SENTELHAS, 1997). O método de Camargo apresentou seus melhores resultados nas estações de verão e outono e seus piores resultados nas estações do inverno e primavera.

Tabela 2. Média, desvio padrão (DP), coeficiente de variação (CV), valor mínimo (Mín) e máximo (Máx) da temperatura média do ar $\left(T_{M E D}\right)$, umidade relativa do ar $(U R)$, velocidade do vento $(V V)$ a dez metros de altura, radiação solar no topo da atmosfera $\left(R_{a}\right)$ e temperatura de ponto de orvalho $\left(T_{d}\right)$, para o período de dez anos (série de 1998 a 2007), nas estações do ano em Curitiba-PR.

\begin{tabular}{|c|c|c|c|c|c|}
\hline$T_{M E D}\left({ }^{\circ} \mathrm{C}\right)$ & Média & DP & CV (\%) & Mín & Máx \\
\hline Verão & 21,3 & 2,2 & 10,3 & 13,8 & 26,1 \\
\hline Outono & 17,1 & 3,5 & 20,5 & 6,3 & 25,4 \\
\hline Inverno & 14,9 & 3,6 & 24,2 & 4,1 & 25,2 \\
\hline Primavera & 18,3 & 3,1 & 16,9 & 8,1 & 26,1 \\
\hline$U R(\%)$ & Média & DP & $\mathrm{CV}(\%)$ & Mín & Máx \\
\hline Verão & 79,2 & 6,8 & 8,6 & 55,0 & 96,7 \\
\hline Outono & 79,7 & 8,1 & 10,2 & 45,8 & 99,0 \\
\hline Inverno & 77,0 & 11,2 & 14,5 & 43,3 & 98,3 \\
\hline Primavera & 79,4 & 8,8 & 11,1 & 47,4 & 97,4 \\
\hline$V V\left(\mathrm{~m} \mathrm{~s}^{-1}\right)$ & Média & DP & CV (\%) & Mín & Máx \\
\hline Verão & 2,1 & 0,6 & 28,6 & 0,6 & 4,6 \\
\hline Outono & 1,9 & 0,7 & 36,8 & 0,4 & 5,6 \\
\hline Inverno & 2,1 & 0,7 & 33,3 & 0,3 & 5,8 \\
\hline Primavera & 2,4 & 1,5 & 25,0 & 1,0 & 25,0 \\
\hline$R_{a}\left(\mathrm{MJ} \mathrm{m}^{-2} \mathrm{dia}^{-1}\right)$ & Média & DP & $\mathrm{CV}(\%)$ & Mín & Máx \\
\hline Verão & 40,4 & 2,7 & 6,7 & 34,3 & 43,3 \\
\hline Outono & 26,1 & 4,2 & 16,1 & 21,0 & 34,3 \\
\hline Inverno & 25,8 & 4,0 & 15,5 & 21,0 & 33,9 \\
\hline Primavera & 40,1 & 2,8 & 7,0 & 33,9 & 43,3 \\
\hline$T_{d}\left({ }^{\circ} \mathrm{C}\right)$ & Média & DP & $\mathrm{CV}(\%)$ & Mín & Máx \\
\hline Verão & 19,1 & 2,1 & 11,0 & 12,3 & 23,4 \\
\hline Outono & 14,8 & 2,1 & 24,3 & 2,1 & 22,5 \\
\hline Inverno & 12,3 & 3,4 & 27,6 & $-2,2$ & 21,2 \\
\hline Primavera & 16,1 & 2,9 & 18,0 & 2,9 & 22,4 \\
\hline
\end{tabular}

Nas condições climáticas de Curitiba-PR, as estimativas da ETo com o método Hargreaves e Samani superestimaram as estimativas realizadas com o método padrão $\left(E T O_{P M}\right)$. Acredita-se que isso ocorreu devido ao clima úmido da região, uma vez que o método de Hargreaves e Samani foi desenvolvido na Califórnia, EUA, em condições semiáridas, a partir de dados obtidos em lisímetros com superfície gramada (PEREIRA et al., 1997). Os resultados corroboram com as observações de Reis et al. (2007) nas regiões norte, sul e serrana do Espírito Santo, que verificaram superestimativas especialmente nas estações chuvosas.

Os valores de $E T o_{B}$ também superestimaram, em média, os valores de EToPM. O método Cultura Agronômica, Ilha Solteira, v.25, n.2, p.155-166, 2016 
relaciona a ETo somente com a temperatura média do ar, sendo indicado apenas em caso de inexistência de dados meteorológicos mais completos ou condições para que se possa estimar a ETo por outro método possuindo melhor embasamento teórico. No presente trabalho, o método de Budyko apresentou resultados mais precisos que o método de Hargreaves e Samani devido ao fato do clima da região ser úmido e, assim, apresentar menor amplitude térmica (MAACK, 2002).

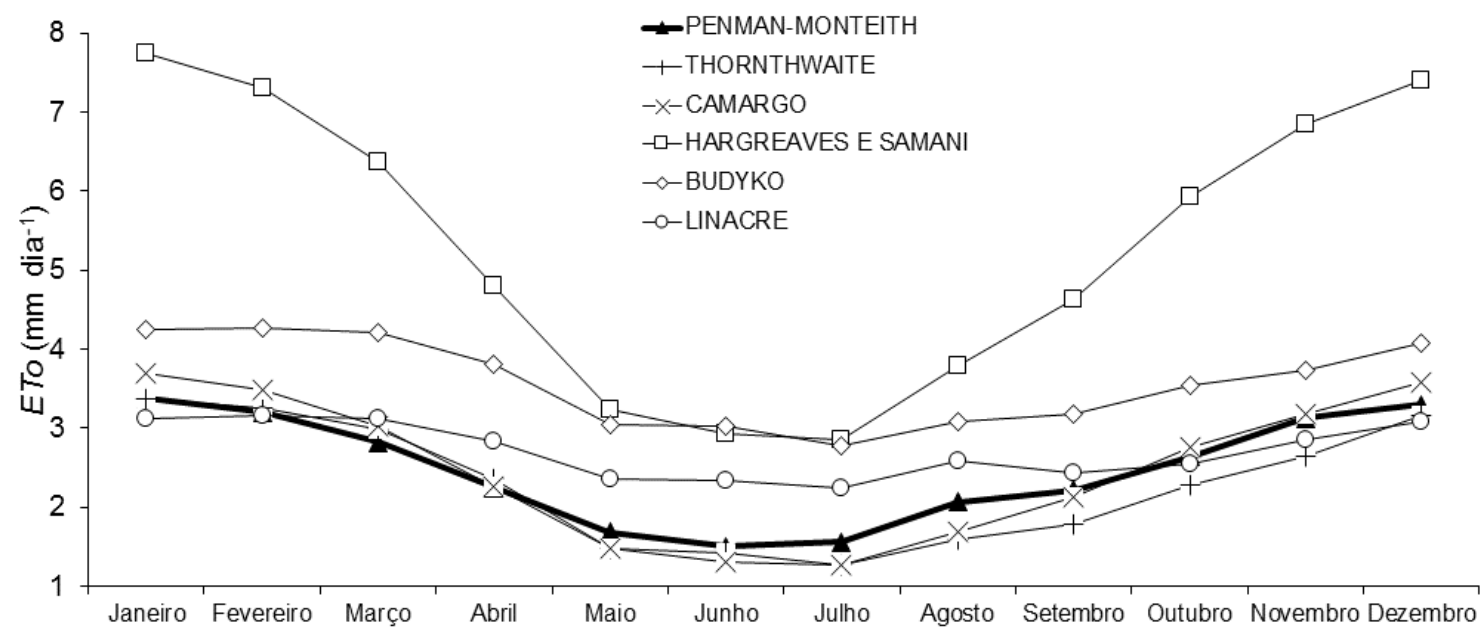

(a)

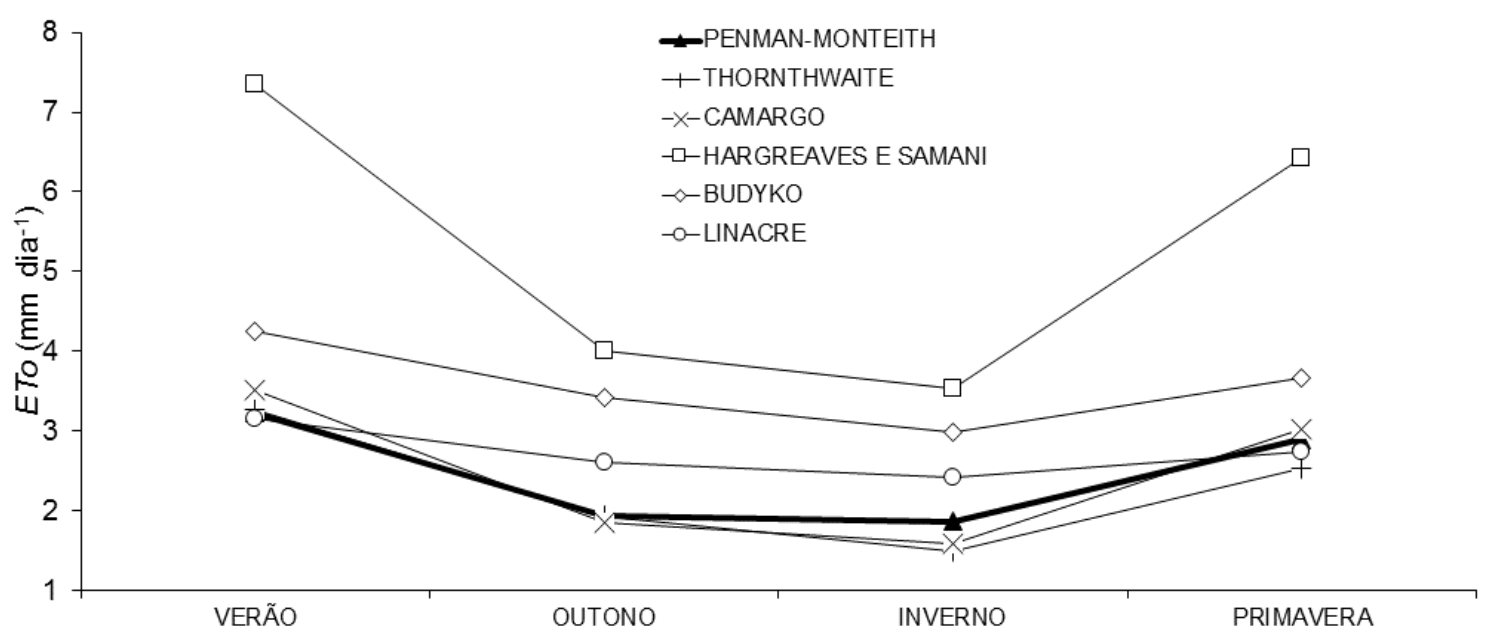

(b)

Figura 1. Estimativa da evapotranspiração de referência média (ETo), obtida no período de dez anos (série de 1998 a 2007) em Curitiba-PR, com os métodos de Penman-Monteith (EToPM), Thornthwaite (EToT), Camargo (EToC), Hargreaves e Samani (ETohs), Budyko $\left(E T o_{B}\right)$ e Linacre $\left(E T o_{L}\right)$ : (a) para os meses do ano; e, (b) para as estações do ano.

O método de Linacre apresentou os melhores resultados para as estações mais quentes (verão e primavera), com tendência a superestimar os valores, principalmente nas estações mais frias (outono e inverno). Como esse método é uma simplificação do método de Penman, no qual utiliza funções da temperatura do ar, como a diferença entre a temperatura média e a temperatura de ponto de orvalho, que foi desenvolvida a partir de dados obtidos em diferentes localidades da África e América do Sul (SOUZA et al., 2014), e para a região de Curitiba- 
PR, a temperatura média do ar é menor, ocasionou um valor de ETo estimado menor.

Os métodos de Thornthwaite $\left(E T o_{T}\right)$, Camargo $\left(E_{T} O_{C}\right)$ e Hargreaves e Samani $\left(\right.$ EToHS $_{\text {) }}$ se destacaram pelos altos valores do coeficiente de correlação. No entanto, analisando o índice de determinação " $d$ ", percebeu-se que apenas os métodos de Thornthwaite e Camargo apresentaram comportamento similar, tendo inclusive o método de Hargreaves e Samani apresentado o pior resultado dentre todos analisados. Os índices de desempenho " $c$ " também mostraram grande similaridade entre os resultados obtidos pelos métodos de Thornthwaite e Camargo (Tabela 3 e Figura 2).

Tabela 3. Coeficiente de correlação (R) e índices " $d$ " de Willmott et al. (1985) e "c" de Camargo e Sentelhas (1997), obtidos para os métodos alternativos de estimativa da evapotranspiração de referência (ETo) tendo o método de Penman-Monteith $($ EToPM) como padrão.

\begin{tabular}{|c|c|c|c|c|c|}
\hline \multirow[b]{2}{*}{ Período } & \multicolumn{5}{|c|}{ Método de estimativa da evapotranspiração de referência (ETo) } \\
\hline & Thornthwaite & Camargo & $\begin{array}{c}\text { Hargreaves e } \\
\text { Samani }\end{array}$ & Budyko & Linacre \\
\hline \multicolumn{6}{|c|}{ Coeficiente de correlação $(\mathrm{R})$ (adimensional) } \\
\hline Anual & 0,85 (muito alta) & 0,89 (muito alta) & 0,86 (muito alta) & 0,77 (muito alta) & 0,62 (alta) \\
\hline Verão & 0,74 (muito alta) & 0,79 (muito alta) & 0,52 (alta) & 0,65 (alta) & 0,66 (alta) \\
\hline Outono & 0,76 (muito alta) & 0,79 (muito alta) & 0,76 (muito alta) & 0,70 (muito alta) & 0,53 (alta) \\
\hline Inverno & 0,71 (muito alta) & 0,72 (muito alta) & 0,73 (muito alta) & 0,62 (alta) & 0,75 (muito alta) \\
\hline Primavera & 0,78 (muito alta) & 0,80 (muito alta) & 0,71 (muito alta) & 0,48 (moderada) & 0,75 (muito alta) \\
\hline \multicolumn{6}{|c|}{ Indices “ $d$ " de Willmott et al. (1985) (adimensional) } \\
\hline Anual & 0,90 & 0,94 & 0,41 & 0,63 & 0,84 \\
\hline Verão & 0,83 & 0,80 & 0,16 & 0,45 & 0,86 \\
\hline Outono & 0,85 & 0,88 & 0,38 & 0,44 & 0,69 \\
\hline Inverno & 0,78 & 0,79 & 0,46 & 0,54 & 0,76 \\
\hline Primavera & 0,81 & 0,88 & 0,26 & 0,64 & 0,90 \\
\hline \multicolumn{6}{|c|}{ Índices de desempenho “c” de Camargo e Sentelhas (1997) (adimensional) } \\
\hline Anual & 0,77 (muito bom) & 0,83 (ótimo) & 0,35 (péssimo) & $0,49(\mathrm{mau})$ & 0,62 (mediano) \\
\hline Verão & 0,62 (mediano) & 0,63 (mediano) & 0,08 (péssimo) & 0,30 (péssimo) & 0,66 (bom) \\
\hline Outono & 0,65 (mediano & 0,70 (bom) & 0,29 (péssimo) & 0,31 (péssimo) & 0,53 (sofrível) \\
\hline Inverno & 0,55 (sofrível) & 0,57 (sofrível) & 0,34 (péssimo) & 0,33 (péssimo) & 0,60 (sofrível) \\
\hline Primavera & 0,63 (mediano) & 0,70 (mediano) & 0,18 (péssimo) & $0,48(\mathrm{mau})$ & 0,75 (bom) \\
\hline \multicolumn{6}{|c|}{ Erro médio absoluto $\left(\mathrm{mm} \mathrm{dia}^{-1}\right)$; Raiz quadrada média do erro ( $\left.\mathrm{mm} \mathrm{dia}^{-1}\right)$} \\
\hline Anual & 0,$41 ; 0,54$ & 0,$33 ; 0,43$ & 2,$86 ; 3,22$ & 1,$13 ; 1,23$ & 0,$49 ; 0,63$ \\
\hline Verão & 0,$33 ; 0,41$ & 0,$34 ; 0,40$ & 4,$14 ; 4,31$ & 1,$04 ; 1,10$ & 0,$31 ; 0,38$ \\
\hline Outono & 0,$35 ; 0,48$ & 0,$26 ; 0,38$ & 2,$07 ; 2,28$ & 1,$50 ; 1,57$ & 0,$68 ; 0,80$ \\
\hline Inverno & 0,$46 ; 0,61$ & 0,$38 ; 0,52$ & 1,$68 ; 1,87$ & 1,$16 ; 1,27$ & 0,$63 ; 0,78$ \\
\hline Primavera & 0,$48 ; 0,62$ & 0,$33 ; 0,41$ & 3,$57 ; 3,78$ & 0,$81 ; 0,89$ & 0,$34 ; 0,41$ \\
\hline
\end{tabular}

O método de Camargo foi o mais adequado para realizar as estimativas da ETo anualmente na região de Curitiba-PR, resultado semelhante foi obtido por Gardiman Junior et al. (2012) para Linhares-ES. Resultados similares foram obtidos por Camargo e Sentelhas (1997) que avaliaram o desempenho de vinte métodos de estimativas da ETo, usando como comparação as medições lisimétricas realizadas no período de 1954 a 1960, em três localidades de São Paulo. Baseando-se no coeficiente $d$ e índice de confiança " $c$ ", os autores obtiveram desempenho muito bom $(0,78$ a 0,91$)$ para os métodos de Thornthwaite e Camargo.

Cultura Agronômica, Ilha Solteira, v.25, n.2, p.155-166, 2016 
Adicionalmente, Syperreck et al. (2008), comparando estimativas da ETo realizadas com métodos alternativos e padrão (Penman-Monteith) na região de Palotina-PR, verificaram também que o método de Camargo apresentou os melhores índices " $c$ " de desempenho.

(a)
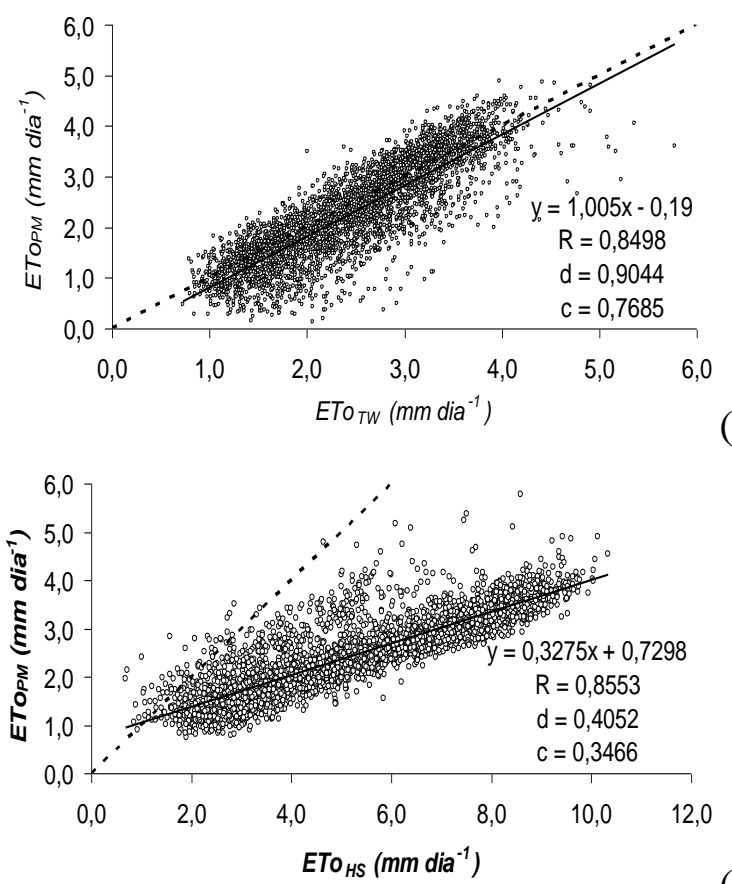

(c)

(b)
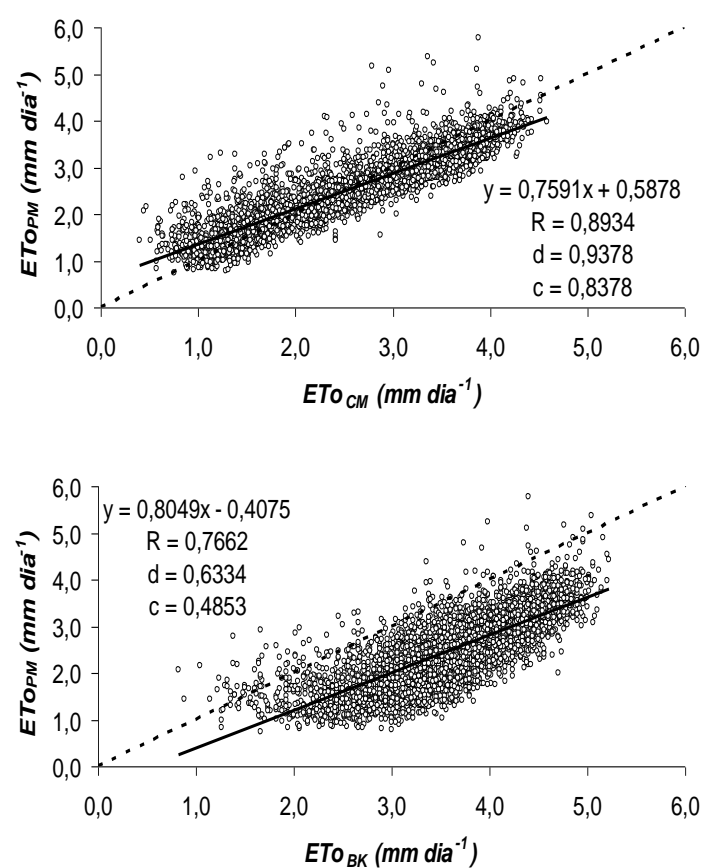

(d)

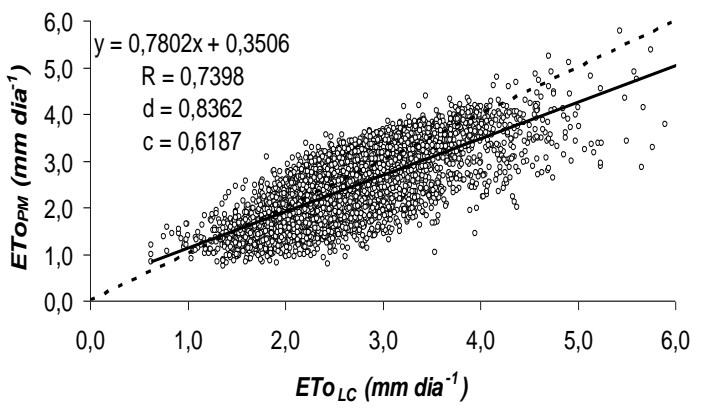

(e)

Figura 2. Análise de regressão linear, coeficiente de correlação (R), índices " $d$ " de Willmott et al. (1985) e "c" de Camargo e Sentelhas (1997), obtidos da relação entre valores diários de Penman-Monteith $\left(E T o_{P M}\right)$ e métodos alternativos Thornthwaite $\left(E T o_{T}\right)$, Camargo $\left(E T o_{C}\right)$, Hargreaves e Samani $\left(E T o_{H S}\right)$, Budyko $\left(E T o_{B}\right)$ e Linacre $\left(E T o_{L}\right)$, considerando os anos de 1998 a 2007, para o período anual.

No entanto, resultados distintos foram obtidos por Conceição (2003), para a Região do Baixo Rio Grande - SP, e Souza et al. (2014), para Nova Venecia - ES e Santa Teresa - ES, nos quais o método de Hargreaves e Samani foi o que apresentou o melhor resultado. Provavelmente em decorrência do tipo climático dessas cidades que possuem classificação Cwa e Aw, respectivamente, porque são climas que possuem temperatura média maior (mais quentes) e a $E T o_{H S}$ considera além da temperatura média, a temperatura máxima do dia.

Apesar disso, Conceição e Mandeli (2005) contestam tal afirmativa porque os autores encontraram no município de Bento Gonçalves, Estado do Rio Grande do Sul, que possui tipo climático Cfa, valores de coeficientes $d$ para o método de Thornthwaite, Camargo, e 
Hargreaves e Samani iguais a 0,79; 0,81; e 0,84, respectivamente, em relação ao método de Penman-Monteith. Já Syperrek et al. (2008) encontraram em Palotina-PR, para os mesmos métodos, valores de 0,$82 ; 0,84$; e 0,85 , respectivamente.

\section{CONCLUSÃO}

O método de Camargo, seguido de Thornthwaite, demonstram melhor desempenho para estimativa anual da evapotranspiração de referência (ETo) em Curitiba-PR, necessitando pequena quantidade de dados de entrada para uma estimativa adequada.

Considerando as estações do ano, os melhores métodos para o verão são Linacre, Camargo e Thornthwaite, para o outono é Camargo, para o inverno são Camargo, Linacre e Thornthwaite, e para a primavera são Linacre e Camargo.

Os métodos de Hargreaves e Samani, e Budyko são inadequados para estimar a ETo para o tipo climático $\mathrm{Cfb}$ na região de Curitiba-PR.

\section{REFERÊNCIAS BIBLIOGRÁFICAS}

ALLEN, R. G.; PEREIRA, L. S.; RAES, D.; SMITH. M. Crop evapotranspiration: guidelines for computing crop water requirements. Rome: FAO, 1998. 297 p.

BUDYKO, M. I. Climate and life. New York: Academic Press, 1974. 508 p.

CAMARGO, A. P. Balanço hídrico no estado de São Paulo. Campinas: IAC, 1971. 28 p.

CAMARGO, A. P.; SENTELHAS, P. C. Avaliação do desempenho de diferentes métodos de estimativa da evapotranspiração potencial no Estado de São Paulo. Revista Brasileira de Agrometeorologia, Santa Maria, v. 5, n. 1, p.89-97, 1997.

COMEC - COORDENAÇÃO DA REGIÃO METROPOLITANA DE CURITIBA. Revista da Região Metropolitana de Curitiba. Curitiba: COMEC, 2015. 89 p.

CONCEIÇÃO, M. A. F. Estimativa da evapotranspiração de referência com base na temperatura do ar para as condições do Baixo Rio Grande, SP. Revista Brasileira de Agrometeorologia, Santa Maria, v. 11, n. 2, p.229-236, 2003.

CONCEIÇÃO, M. A. F.; MANDELLI, F. Comparação entre métodos de estimativa da evapotranspiração de referência em Bento Gonçalves, RS. Revista Brasileira de Agrometeorologia, Santa Maria, v. 13, n. 2, p.303-307, 2005.

GARDIMAN JUNIOR, B. S.; MAGALHÃES, I. A. L.; CECILIO, R. A. Comparação entre diferentes métodos de estimativas de evapotranspiração de referência (ETo) para LinharesES. Nucleus, Ituverava, v. 9, n. 2, p.103-112, 2012.

HARGREAVES, G. H.; SAMANI, Z. A. Reference crop evapotranspiration from temperature. Applied Engineering Agriculture, Saint Joseph, v. 1, n. 2, p.96-99, 1985.

Cultura Agronômica, Ilha Solteira, v.25, n.2, p.155-166, 2016 
HOPKINS, W. G. Correlation coefficient: a new view of statistics. 2000. Disponível em: http://www.sportsci.org/resource/stats/correl.html . Acesso em: 01 abr. 2016.

LINACRE, E. T. A simple formula for estimating evapotranspiration rates in various climates, using temperature data alone. Agricultural Meteorology, Amsterdam, v. 18, n. 6, p.409-424, 1977.

MAACK, R. Geografia física do estado do Paraná. 3. ed. Curitiba: Imprensa Oficial, 2002. $709 \mathrm{p}$.

MAPA - MINISTÉRIO DA AGRICULTURA, PECUÁRIA E ABASTECIMENTO.

Normais climatológicas (1961-1990). Brasília: Secretaria Nacional de Irrigação/Departamento Nacional de Meteorologia, 1992. 54 p.

PEREIRA, A. R.; VILA NOVA, N. A.; SEDYAMA, G. C. Evapo(transpi)ração. Piracicaba: ESALQ, 1997. $183 \mathrm{p}$.

PRAVEENA, S. M.; ABDULLAH, M. H.; BIDIN, K.; ARIS, A. Z. Modeling of water balance components in a small island via a numerical model application. Journal of Coastal Research, Coconut Creek, v. 28, n. 1, p. 202-209, 2012.

REIS, E. F.; BRAGANÇA, R.; GARCIA, G. O.; PEZZOPANE, J. E. M.; TAGLIAFERRE, C. Estudo comparativo da estimativa da evapotranspiração de referência para três localidades do Espírito Santo no período seco. IDESIA, Tarapacá, v. 25, n. 3, p.75-84, 2007.

SEDIYAMA, G. C. Necessidade de água para os cultivos. Brasília: ABEAS, 1987. 145 p.

SOUZA, J. L. M.; VILLA NOVA, N. A.; CASTRO NETO, P. Avaliação do efeito da energia advectiva na estimativa da evapotranspiração de referência para a região de Lavras/MG. Revista do Setor de Ciências Agrárias, Botucatu, v. 13, n. 1-2, p.41-47, 1994.

SOUZA, J. M.; PEREIRA, L. R.; RAFAEL, A. M.; SILVA, L. D.; REIS, E. F.; BONOMO, $\mathrm{R}$. Comparison of methods for estimating reference evapotranspiration in two locations of Espirito Santo. Revista Brasileira de Agricultura Irrigada, Fortaleza, v. 8, n. 2, p.114-126, 2014.

SYPERRECK, V. L. G.; KLOSOWSKI, E. S.; GRECO, M.; FURLANETTO, C. Avaliação de desempenho de modelos para estimativas de evapotranspiração de referência para a região de Palotina, Estado do Paraná. Acta Scientiarum Agronomy, Maringá, v. 30, n. 5, p.603609, 2008.

THORNTHWAITE, C. W. An approach toward a rational classification of climate. Geographical Review, New York, v. 38, n. 1. p.55-94, 1948.

WILLMOTT, C. J.; ROWE, C. M.; MINTZ, Y. Climatology of terrestrial seasonal water cicle. Journal of Climatology, Malden, v. 5, n. 1, p.589-606, 1985.

XU, C. Y.; SINGH, V. P. Evaluation of three complementary relationship evapotranspiration models by water balance approach to estimate actual regional evapotranspiration in different climatic regions. Journal of Hydrology, New York, v. 308, n. 1-4, p.105-121, 2005.

Cultura Agronômica, Ilha Solteira, v.25, n.2, p.155-166, 2016 